\title{
Non-volatile analysis in fruits by laser resonant ionization spectrometry: application to resveratrol $\left(3,5,4^{\prime}\right.$-trihydroxystilbene $)$ in grapes
}

\author{
C. Montero, J.M. Orea, M. Soledad Muñoz, R.F.M. Lobo*, A. González Ureña** \\ Unidad de Láseres y Haces Moleculares, Instituto Pluridisciplinar, Universidad Complutense de Madrid, P Juan XXIII, 1, 28040 Madrid, Spain \\ Received: 29 March 2000/Revised version: 23 May 2000/Published online: 16 August 2000 - (C) Springer-Verlag 2000
}

\begin{abstract}
A laser desorption (LD) coupled with resonanceenhanced multiphoton ionisation (REMPI) and time-of-flight mass spectrometry (TOFMS) technique for non-volatile trace analysis compounds is presented. Essential features are: (a) an enhanced desorption yield due to the mixing of metal powder with the analyte in the sample preparation, (b) a high resolution, great sensitivity and low detection limit due to laser resonant ionisation and mass spectrometry detection. Application to resveratrol content in grapes demonstrated the capability of the analytical method with a sensitivity of $0.2 \mathrm{pg}$ per single laser shot and a detection limit of $5 \mathrm{ppb}$.
\end{abstract}

PACS: 42.62.Fi; 82.80.Ms

One of the crucial problems in the development of new analytical methods, specially in the case of food samples, is the detection and identification of non-volatile organic compounds present at low concentration levels. Although mass spectrometry nowadays is widely used in the analysis of such compounds providing exact mass identification, the difficulty lies in their volatilisation into the gas phase prior to injection into the analyser. This first-step requirement is particularly a problem for thermally labile samples as they rapidly decompose upon heating.

To circumvent this difficulty a wide range of techniques have been developed for non-volatile analysis. Namely: fast atom bombardment (FAB) [1], field desorption (FD) [2], laser desorption (LD) [3], plasma desorption mass spectrometry (PDMS) [4] and secondary ion mass spectrometry (SIMS) [5]. In spite of the significant improvement and extended applicability of these methods they all suffer of the same limitation derived from the fact that both desorption and ionisation cannot be separately optimised, which, obviously, may be critical for many real applications. Nevertheless the

\footnotetext{
*Present address: Departamento de Fisica. Facultade de Ciencias e Tecnologia. Universidade Nova de Lisboa. Quinta da Torre. 28025 Monte da Caparica, Portugal

** Corresponding author.

(Fax: +34-91/394-3265, E-mail: laseres@eucmax.sim.ucm.es)
}

matrix-assisted laser desorption/ionisation (MALDI) technique is widely used for ion generation in many mass spectrometric analytical applications [6]. This is accomplished by mixing the analyte with an excess of cromophore matrix so as to improve both the desorption and ionisation processes, especially in the case of protein and polymer analysis. However, due to the complexity of the process, finding the best cromophore or matrix for a particular analyte is not always straightforward. A significant improvement using the MALDI technique was obtained by the so-called ultra fine metal plus liquid matrix method (UFP) for sample preparation [7]. This procedure increases the yield of high-mass ions and prolongs their production time.

More recently, laser desorption methods have been developed in which volatilisation and ionisation steps are separated and so higher sample sensitivity is achieved. While all them have in common the laser desorption step they differ in their ionisation method. Within this category the laser multiphoton ionisation coupled with time-of-flight mass spectrometry, and in particular resonance-enhanced multiphoton ionisation (REMPI)-time-of-flight mass spectrometry (TOFMS) can be considered as one of the most powerful methods for the analysis of trace components in complex matrix [8-17].

The high selectivity of REMPI-TOFMS is given by the combination of the mass selective detection with the resonant ionisation process: the ionisation is produced by the successive absorption of two or more laser photons. Thus for an efficient ionisation, the energy of the first photon has to be resonant with one of the real electronic states in the molecule (resonance enhancement). This condition gives a second selectivity to the technique: laser wavelength-selective ionisation. In addition, other clear advantages of REMPI-TOFMS are: the great sensitivity and resolution, a major ionisation efficiency, an easy control of the molecular fragmentation by the laser intensity and the possibility of simultaneous analysis of different components present in a matrix. As a result this technique is well established for spectroscopic analysis, either for fundamental research or for applications where very sensitive analysis of trace components in a complex matrix or simultaneous analysis of a large number of components over a range of concentrations are needed. 
In spite of several theoretical studies done in this field [18], the mechanism for LD is still not well understood This is mainly due to the complexity of the processes involved in laser desorption, i.e. the excitation of the absorbing molecules, the energy transfer from the excited molecules in the internal and translational modes of the substrate, the plume development with its characteristic velocity and angle, the energy cooling of the molecules due to the expansion in vacuum if it is the case, etc.

In this communication a simple technique to enhance the desorption yield is presented. As in MALDI the enhancement is accomplished by mixing the analyte with an adequate matrix but here LD is combined with resonance-enhanced multiphoton ionisation (REMPI) and time-of-flight mass spectrometry (TOFMS) and the matrix is only used to enhance the neutral desorption. The matrix consists of metal powder which resulted in significant enhancement of the desorption yield. The desorption and ionisation processes remain physically separated, so that both can be independently optimised. The method has been developed in order to find the analytical requirements necessary for the determination of resveratrol in grapes, and the first results are also presented.

Trans-resveratrol $\left(3,5,4^{\prime}\right.$-trihydroxystilbene) is an antioxidant compound naturally produced in a huge number of plants, including grapes, as phytoalexin, i.e. an antimicrobial or antifungal agent produced by the plant upon infection. Resveratrol is mainly located in the plant leaves [19] and grape skin [20] and has been found in wines in concentrations depending on viticultural and enological practices [21,22].

\section{Experimental}

The experimental set-up has been already described elsewhere [23], so only a brief description is given here. Essentially, it consists of two independent high vacuum chambers; the first chamber is used for both laser desorption and laser post-ionisation of the sample followed by the ions acceleration towards the second chamber, basically a linear time-offlight unit with a dual microchannel plate detector.

The analyte is deposited by spraying the solution on a pyrex disc which is parallel to the first accelerating plate. A step motor is used to control the disc rotation. The whole sample holder and motor assembly is attached to a linear translator so that adjustment of the perpendicular direction to the desorption laser can be made. This movement changes the rotation radius so as to increase the number of runs for a given sample.

Pulses of $5 \mathrm{~ns}$ of duration of the second harmonic of a Nd:YAG laser were used for sample desorption and a frequency-doubled dye laser was used to ionise the desorbed neutrals. As discussed below (Sect. 3) to estimate the amount of desorbed resveratrol the desorption area has to be known. This is done by collimating the desorption laser and a careful measurement of the laser spot, i.e. illuminated area right at the desorption plate. For laser spot sizes of the order of $12.5 \mathrm{~mm}^{2}$ the error was of the order of $8 \%$ to $10 \%$. The ionisation laser was always perpendicular to that of desorption and crossed the desorbed particles at a variable distance ranged from 6 up to $30 \mathrm{~mm}$ apart from the desorption zone. Finally, a system for frequency-doubling the dye laser output and active wavelength laser scanning (INRAD-AT-III-UV) with a doubling crystal (BBO-TST) allowed us to scan the output from 235 up to $365 \mathrm{~nm}$.

The ions produced by the second laser pulse are accelerated towards the TOF tube by a Whiley-McLaren-type acceleration system and detected by a two-microchannel-plates detector (COMSTOCK CP-625C/50F) placed at the end of the TOF tube.

In order to have a first approximation to the wavelength region where the compound to be analysed shows resonant absorption, it is important to know its UV absorption spectrum which is obtained by means of a JASCO Model V-530 UV-VIS spectrophotometer.

\section{Sample preparation}

A basic feature of the technique is the absence of any separation and/or extraction method for sample preparation, i.e. the sample is used directly as described below. Figure 1 shows a pictorial view of the sample deposition device. The sample (i.e. pepper, grapes, etc.) is triturated and homogenised by means of an Ultra-Turrax T25 IKA mixer, allowing us to obtain a final dispersion from 5 up to $25 \mu \mathrm{m}$. A suitable solvent (generally acetone) is added as carrier. The grinding is done

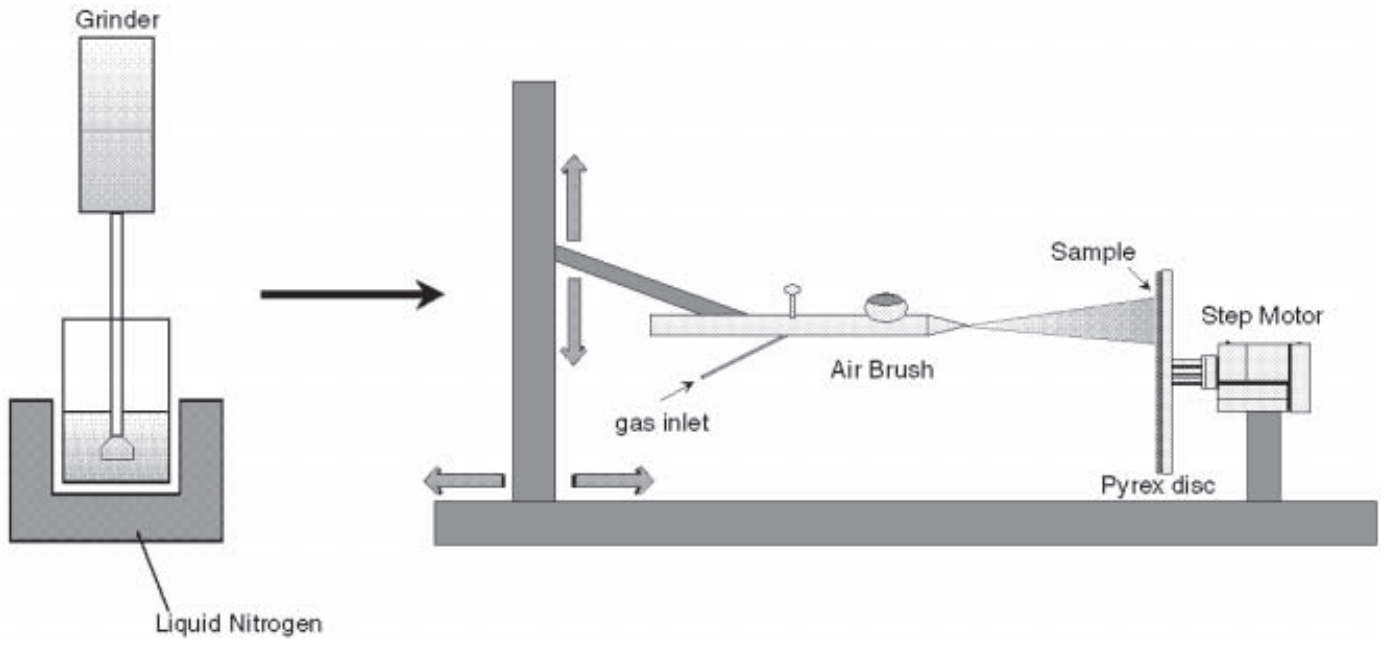

Fig. 1. Pictorial view of the sample deposition device (see text for details) 
by cooling the mixture with liquid $\mathrm{N}_{2}$ in order to increase the breaking efficiency of the sample particles and hence to ensure a better dispersion. After grinding, the suspension is sprayed on a Pyrex disc by means of an air-controlled spray. To get a better homogeneity the Pyrex disc is attached to a rotating step motor during the operation.

\section{Results and discussion}

\subsection{Spectroscopic studies: multiphoton laser ionisation}

One interesting parameter to be determined is the photoionization order of the molecule under analysis. In a photoionization process, obtained by a monochromatic non-resonant photon flux of intensity $\Phi$ with only one electron released per ionisation event, the intensity of the detected ion signal can be related to the photon flux as: $\mathrm{I} \sim \Phi^{n}$ where $n$ is the order of the photoionization process, i.e. the number of photons necessary to reach the ionisation threshold. As the photon flux is proportional to the laser beam energy, $E$, the slope of the log-log plot of the ions signal intensity versus the laser energy, gives the effective order of photoionization. For resveratrol the variation of the signal with the ionisation energy was investigated using the frequency-doubled dye laser output at a wavelength of $299.5 \mathrm{~nm}$, with energies between 80 and $270 \mu \mathrm{J} /$ pulse, and a laser desorption energy fixed at $13 \mathrm{~mJ} /$ pulse. The off-resonance 299.5-nm wavelength was specifically selected to avoid saturation effects. Figure 2 shows a log-log plot of the ion signal intensity versus the ionisation energy; a clear signal enhancement with the laser energy is noticeable, and the value obtained for the slope $(1.9 \pm 0.2)$ suggests that resveratrol is ionised through a onecolour two-photons ionisation (R2PI) process. To check that saturation effects play little role, if any, in the results shown in Fig. 2 different runs were made at lower ionisation energies. All them resulted in the same (about 2) slope in the log-log plot.

The main prerequisite for resonance-enhanced mass spectrometric analysis is the knowledge of the resolved REMPI spectrum of the compound to be analysed, so as to use the

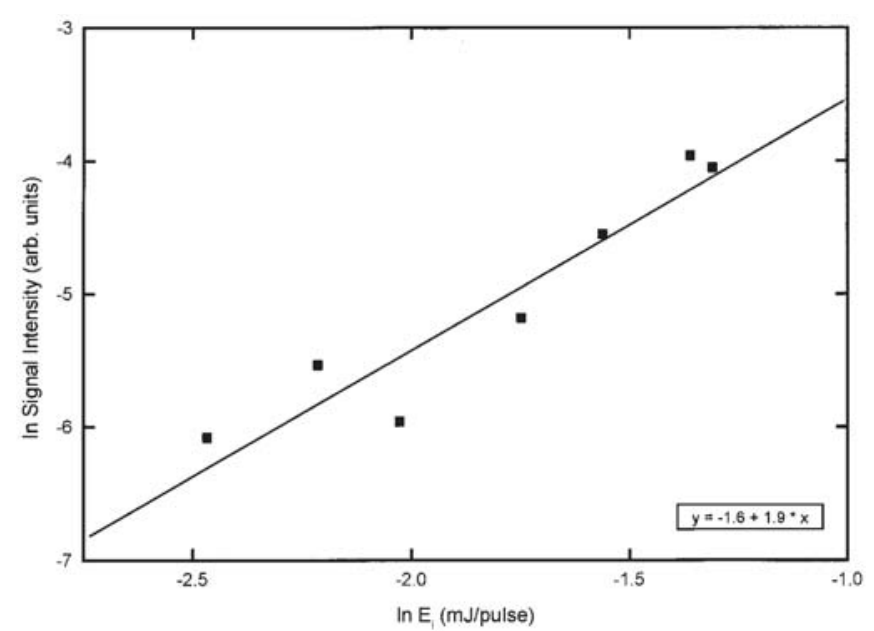

Fig. 2. Log-log plot of the resveratrol signal vs. the ionisation laser energy at $299.5 \mathrm{~nm}$. The inset shows a linear regression fit of the data suggesting a one-colour, two-photon (R2PI) process optimal wavelength for selective ionisation. Thus the tuneable dye laser allows us to use photons of resonant energy to produce a transition between real electronic states of the molecule under study, and hence to involve a moleculespecific parameter in the ionisation process.

The UV absorption spectrum of trans-resveratrol shows a wide band from 280 to $360 \mathrm{~nm}$ with the maximum of absorbance at $306 \mathrm{~nm}$. In order to find the REMPI spectrum for trans-resveratrol, the wavelength of the frequencydoubled dye laser output was tuned between these two values. We could then determine that the wavelength region where resveratrol shows resonant ionisation ranges from 301.5 up to $307.5 \mathrm{~nm}$ with the maximum at $302.1 \mathrm{~nm}$ [24] which is then selected as the optimal wavelength for resveratrol analysis in complex samples.

\subsection{Metal powder enhanced desorption (MEPED)}

For resveratrol analysis several methods have been tested in order to improve the desorption yield and hence the detection limit of the technique. As mentioned above, in MALDI one mixes the analyte with an excess of cromophore matrix to improve both the desorption and ionisation processes, specially in the case of large, non-volatile and thermally labile molecules. In the present work, the matrix is only used to enhance the first step, i.e. the neutral desorption, maintaining as stated above, the ionisation physically separated from it, so that both desorption and ionisation can be independently optimised.

As is well known, Rhodamine dyes have been commonly used in laser desorption experiments. Since the desorption wavelength used in these experiments is $532 \mathrm{~nm}$, Rhodamine 590 seemed to be a good candidate in order to optimise the desorption process because it shows the absorption maximum at $530 \mathrm{~nm}$. Nevertheless the enhancement factor of the resveratrol signal obtained with Rhodamine mixture (see below) appeared not to be enough for our analytical requirements. A considerable improvement of the desorption yield was found by adding $\mathrm{Zn}$ powder of about $10 \mu \mathrm{m}$ particle diameter to the analyte.

Figure 3 (top) displays the laser resonant ionisation timeof-flight spectrum of pure resveratrol obtained with a desorption energy of $50 \mathrm{~mJ} /$ pulse and an ionisation energy of $254 \mu \mathrm{J} /$ pulse at $302.1 \mathrm{~nm}$; the signal corresponds to a maximum of $2.13 \mu \mathrm{g}$ of resveratrol, which is the quantity present in the desorption area (taken as the laser illuminated area, see above) and hence desorbed assuming that the laser completely desorbs the sample in this irradiated area. The middle figure shows the spectrum obtained with the same quantity of resveratrol and experimental conditions except for the use of Rhodamine 590 (0.17 mg in the desorption area). The bottom spectrum corresponds to the same quantity of resveratrol with $\mathrm{Zn}$ powder $(0.02 \mathrm{mg}$ in the desorption area) and the rest of conditions unchanged. It should be noted that in order to compare both experiments the quantities of Rhodamine 590 and $\mathrm{Zn}$ powder added to the analyte are equimolars. In Fig. 3, middle and bottom spectra have been moved down for clarity.

From the comparison of Fig. 3 results the significant signal enhancement when using the metal powder enhanced desorption (MEPED) technique is clearly noticeable. Whereas with Rhodamine 590 the resveratrol signal is enhanced by 


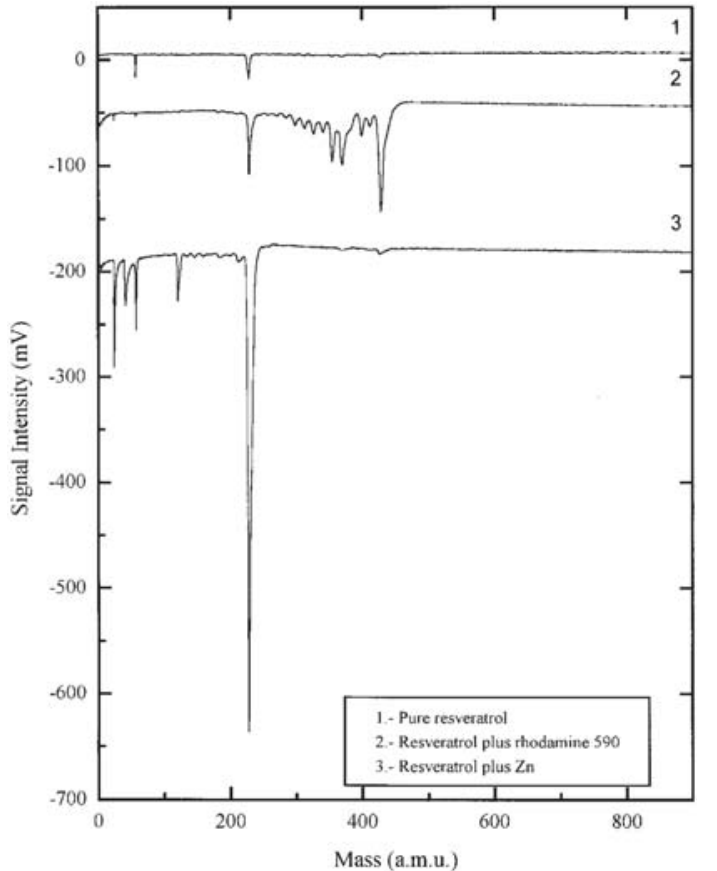

Fig. 3. (Top) TOF mass spectrum of pure resveratrol obtained with a desorption energy of $50 \mathrm{~mJ} /$ pulse and a ionisation energy of $245 \mu \mathrm{J} /$ pulse at $\lambda$ ionisation $=302.1 \mathrm{~nm}$. (Middle) Same as the top spectrum but with $0.17 \mathrm{mg}$ of Rhodamine 590 added. (Bottom) Same as the top spectrum but with $0.02 \mathrm{mg}$ of $\mathrm{Zn}$ powder added. The middle and bottom spectra have been shifted for a better display. Notice the 20 -fold enhancement factor in the bottom spectrum

a factor of 2.5 with respect to the pure resveratrol, a 20-fold enhancement factor is obtained by adding $\mathrm{Zn}$ powder.

Besides this signal enhancement, another important feature of the MEPED technique is an approximately 10 times better signal-to-noise ratio that enables a higher detection limit in our analysis, a basic objective of this study. As is well known, the detection limit of a method is the lowest analyte concentration that produces a response detectable above the noise level of the system, and generally this is assumed as three times the noise level. Consequently the MEPED sample preparation method provides a 10 times lower detection limit as compared with the pure resveratrol spectrum. The ten-fold increase in the detection limit obtained for the MEPED technique should be taken as an upper limit, as the observed signal enhancement does not neccesarily mean the same increase in sensitivity. Indeed part of the signal enhancement is due to the larger "effective" area of desorption because of a better heat conduction when the $\mathrm{Zn}$ metal powder is present. At the present stage we have no quantitative method to account for this effect and correct its influence in the improvement of the detection limit. Work will be carried out in our laboratory using the same $\mathrm{Zn}$ powder mixture with different particle diameters such that further information could be gained to account for this uncertainty.

The underlying physical mechanism responsible for the enhancement in the resveratrol desorption yield associated with the presence of the $\mathrm{Zn}$ powder is not yet fully understood. Nevertheless, some qualitative arguments can be considered. Clearly the coupling between the metal electrons and the intense electrical field of the laser radiation ensures a fast and efficient local heating of the metal substrate. Further- more, the increased effective area of the $10-\mu$ m-diameter $\mathrm{Zn}$ powder strongly facilitates the metal desorption and, consequently, that of adsorbed or surrounding analyte. As a result, the combination of a fast laser-induced desorption, favoured by the electron metal heating, increased effective area, with a facile ablation of the (micrometer) metallic components of the matrix contribute to an increase in the desorption yield of a factor of 20 with respect to that observed in the pure resveratrol. It is also interesting to compare the MEPED yield even with that of Rhodamine mixture. Here it is very likely that rhodamine clusters may be present even with an effective diameter smaller than $10 \mu \mathrm{m}$. Therefore, the distinctive feature from MEPED is the absence of delocalized electrons, i.e. its non-metallic character, that may preclude the fast and efficient local heating, albeit resonant absorption wavelength (i.e. $532 \mathrm{~nm}$ ) is used. As a result the latter feature plus the presence of significant fragmentation, shown in the TOF spectrum, probably due to multiphoton absorption of the parent molecule make the resveratrol desorption yield a less efficient process than in MEPED.

The volatility enhancement of the sample due to "rapid heating" was also suggested in the UPF method in which an improvement of an order of magnitude in precision was reported. A clear advantage of the present MEPED method with respect to UFP is the separate desorption and ionisation steps in the former. This allows an optimisation of the neutral desorption prior to the ionisation. Furthermore, the use of selective resonant ionisation in the second step ensures both a higher resolution and a lower detection limit.

\subsection{Analytical applications}

Analytical interest in resveratrol was first due to its natural pesticide character and hence its ability to improve the natural resistance of plants to fungal infections. But several subsequent investigations demonstrate that human consumption of resveratrol could have many health benefits, namely to decrease lipid deposition in liver [25], to inhibit blood platelet aggregation [26], the oxidation of

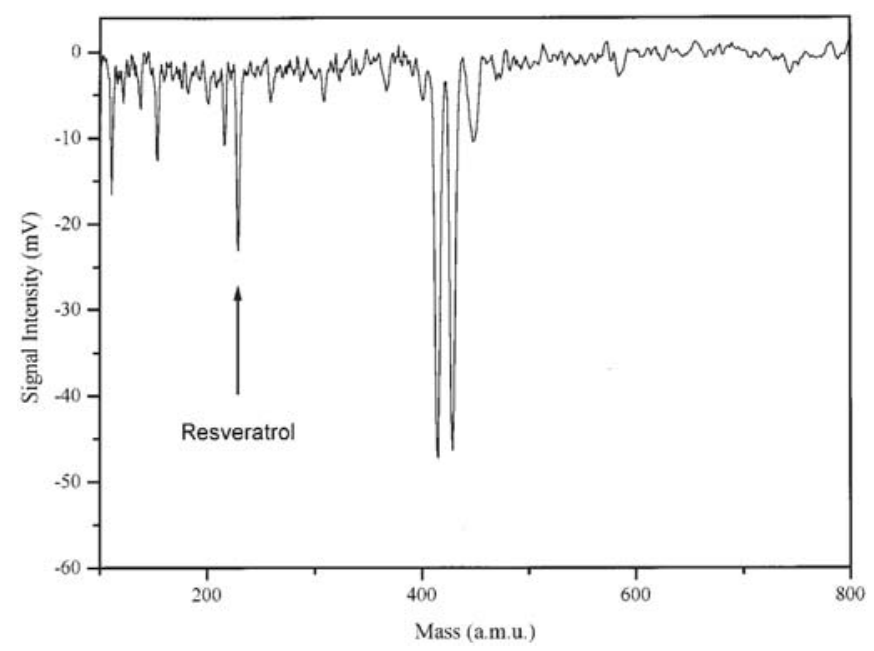

Fig. 4. TOFMS spectrum of a grape skin sample obtained at the laser conditions shown in the inset. A signal-to-noise ratio of $R=12.6$ leads to a detection limit of about 5 ppb of resveratrol in grape skin 
low-density lipoproteines [27], and to protect against cardiovascular diseases [28]. Recently the cancer chemopreventive activity of resveratrol in assays representing the three major stages of carcinogenesis: initiation, promotion and progression [29] was reported attracting a considerable attention.

Figure 4 shows a time-of-flight spectrum obtained using the MEPED technique from a sample of white grape skin whose resveratrol content is already known [30] to be $0.020 \mu \mathrm{g}$ of resveratrol/g of skin, i.e. $20 \mathrm{ppb}$ of resveratrol in grape skin. The displayed spectrum corresponds to a desorption area with $48 \mu \mathrm{g}$ of grape skin and $79 \mu \mathrm{g}$ of $\mathrm{Zn}$, i.e. to about $1 \mathrm{pg}$ of resveratrol. A rough stimate of the detection limit from this spectrum resulted in $5 \mathrm{ppb}$ of resveratrol in grape skin.

As mentioned above, most of the bibliographic sources indicate that resveratrol is mainly accumulated in the grape skin, so we carried out a separate analysis of a sample containing only the grape flesh and seeds, in order to investigate the resveratrol content in this part of the berry. The time-offlight spectrum obtained at the same experimental conditions from this sample showed no significant signal of resveratrol, proving that the main content of resveratrol in grapes is accumulated in the skin.

\section{Concluding remarks}

An analytical technique based on laser desorption coupled with REMPI-TOFMS to allow fast, direct and high sensitive probing of trans-resveratrol in complex samples (specially plants and fruits) has been developed. First of all, laser desorption was shown to be a convenient method to solve one of the long-standing needs in non-volatile analysis: that is direct sample preparation. Second, the MEPED method for sample preparation presented here showed a 20-fold enhancement factor in the desorption yield and a better signal-to-noise ratio, which improved the detection limit for resveratrol analysis. This enhancement factor was attributed to an increase in the heating speed of the sample due to (a) the coupling between the electric field of the laser photon and the delocalized electrons of the added metal powder and (b) the increased surface area resulted from the use of $10 \mu \mathrm{m}$ particle size. Finally the great sensitivity and resolution arising from the use of selective ionisation and mass spectrometry are of major relevance and should be remarked on.

As mentioned, the reported method demonstrated its capability for the detection of resveratrol in grapes. The main features are a detection limit of about $5 \mathrm{ppb}$ of trans-resveratrol in grape skin and a sensitivity in the order of $0.2 \mathrm{pg}$ per single laser shot which we think mark the current state of the technique for thermally labile and non-volatile chemical analysis. Work is now in progress for the validation of the technique (i.e. linearity, detection and quantification limits, reproducibility, accuracy, etc.) aimed at developing a timely, accurate and reliable method for quantitative analysis not only of trans-resveratrol in grapes, but also for other nonvolatile present in agricultural samples.

Acknowledgements. This work received financial support from the European Union project number FAIR-CT98-4211 and the Comunidad Autónoma de Madrid. J.M.O. acknowledges his postdoctoral contract to the Ministerio de Educación y Ciencia of Spain. R.F.M. L. acknowledges the financial assistence from FCT-ICCTI (Portugal).

\section{References}

1. J.E. Campana, R.B. Fress: J. Chem. Soc., Chem. Commun. 21, 1414 (1984)

2. G.D. Daves, Jr.: Acc. Chem. Res. 12, 359 (1979)

3. E.D. Hardin, T.P. Fan, C.R. Blakely, M.L. Vestal: Anal. Chem. 56, 2 (1984)

4. R.D. MacFarlane, D. Vemura, K. Veda, Y. Hirata: J. Am. Chem. Soc. 102, $875(1980)$

5. A. Benninghoven, W. Sichtermann: Anal. Chem. 50, 1180 (1978)

6. M. Karas, F. Hillenkamp: Anal. Chem. 60, 2299 (1988)

7. K. Tanaka, H. Waki, Y. Ido, S. Akita, Y. Yoshida, T. Yoshida: Rapid Commun. Mass Spectrom. 2, 151 (1988)

8. R.N. Zare: Science 226, 298 (1984)

9. B. Bescós, J.M. Orea, C. Montero, A. González Ureña, C. Weickhardt, U. Boels, E.W. Schlag: Chem. Phys. Lett. 287, 371 (1998)

10. M.N. Al-Kahali, R.J. Donovan, T. Ridley: Chem. Phys.208, 165 (1996)

11. J.B. Milan, W.J. Buma, M.N.R. Ashfold: Chem. Phys. Lett. 239, 326 (1995)

12. J.C. Polanyi, N.S.-K. Sze, J.-X. Wang: J. Phys. Chem. 101, 6679 (1997)

13. G.K. Nicolussi, M.J. Pellin, W.F. Calaway, R.S. Lewis, A.M. Davis, S. Amari, R.N. Clayton: Anal. Chem. 69, 1140 (1997)

14. C.M. Gittins, M.J. Castaldi, S.M. Senkan, E.A. Rohlfing: Anal. Chem. 69, 286 (1997)

15. C. Weickhardt, C. Grun, R. Heinicke, A. Meffert, J. Grotenmeyer: Rapid Commun. Mass Spectrom. 11, 745 (1997)

16. Zimmerman, U. Boels, H.J. Heger, E.R. Rohwer, E.K. Ortner, E.W. Schlag, A. Kettrup: J. High Res. Chrom. 20, 461 (1997)

17. O.P. Haefliger, R. Zenobi: Anal. Chem. 70, 2660 (1998)

18. L.V. Zhigilei, P.B.S. Kodali, B.J. Garrison: J. Phys. Chem. B 102, 2845 (1998)

19. R. Pezet, P. Cuenat: Am. J. Enol. Vitic. 47, 287 (1996)

20. A.L. Waterhouse, R.M. Lamuela Raventós: Phytochemistry 37, 571 (1994)

21. U. Vrhovsek, S. Wendwlin, R. Eder: Am. J. Enol. Vitic. 48, 214 (1997)

22. G.J. Soleas, J. Dam, M. Carey, D.M. Goldberg: J. Agric. Food Chem. 45, 3871 (1997)

23. J.M. Orea, B. Bescós, C. Montero, A. González Ureña: Anal. Chem 70, 491 (1998)

24. C. Montero, B. Bescós, J.M. Orea, A. González Ureña: Reviews in Analytical Chemistry 19, 1 (2000)

25. Y. Kimura, H. Ohminami, H. Okuda, K. Baba, M. Kozawa, S. Arichi: Planta Med. 49, 51 (1983)

26. A.A.E. Bertelli, L. Giovannini, D. Giannessi, M. Migliori, W. Bernini, M. Fregoni, A.J. Bertelli: Tissue React. 17, 1 (1995)

27. E.N. Frankel, A.L. Waterhouse, J.E. Kinsella: Lancet 341, 1103 (1993)

28. M.H. Criqui: Lancet 8939/8940, 1719 (1994)

29. M. Jang, L. Cai, G.O. Udeani, K.V. Slowing, C.F. Thomas, C.W.W. Beecher, H.H.S. Fong, N.R. Farnsworth, A.D. Kinghorn, R.G. Mehta, R.C. Moon, J.M. Pezzuto: Science 275, 218 (1997)

30. Unpublished results from this laboratory 